\title{
Dynamics of the Open Cluster NGC 188: A Comparison to an $N$-body Simulation of M67
}

\author{
Aaron M. Geller ${ }^{1}$, Robert D. Mathieu ${ }^{1}$, Hugh C. Harris ${ }^{2}$ and Robert \\ D. McClure ${ }^{3}$ \\ ${ }^{1}$ Department of Astronomy, University of Wisconsin - Madison, WI, 53706, USA; \\ geller@astro.wisc.edu \\ ${ }^{2}$ United States Naval Observatory, Flagstaff, AZ, 86001, USA \\ ${ }^{3}$ Dominion Astrophysical Observatory, Victoria, B.C., V8X 4M6, Canada
}

\begin{abstract}
We present a detailed dynamical study of the old (7 Gyr) open cluster NGC 188. Our combined radial-velocity data set spans a baseline of 35 years, a magnitude range of $12 \leqslant \mathrm{~V} \leqslant 16.5$, and a $1^{\circ}$ diameter region on the sky. Our magnitude limits include solar-mass main-sequence stars, subgiants, giants, and blue stragglers, and our spatial coverage extends radially to 11.5 core radii. We have measured radial velocities for 1014 stars in the direction of NGC 188 with a precision of $0.4 \mathrm{~km} \mathrm{~s}^{-1}$, and have calculated radial-velocity membership probabilities for stars with $\geqslant 3$ measurements. We find 420 stars to be high-probability cluster members, including 137 spectroscopic binaries. These detectable binaries all have orbital periods of less than $10^{4}$ days, and thus are hard. We have derived orbit solutions for 67 member binary stars, and use our 35 main-sequence binaries with orbit solutions to compare the eccentricity and period distributions with simulated observations of the Hurley et al. (2005) model of M67 (4.5 Gyr). We also compare the spatial distributions of cluster member populations.
\end{abstract}

Keywords. Stars: spectroscopic binaries, blue stragglers, Galaxy: open clusters

\section{Introduction}

We use precise radial-velocity (RV) observations of the old (7 Gyr) open cluster NGC 188 to perform a detailed study of the hard binary population and the spatial distribution of cluster member populations. We then use these results to conduct the first in-depth comparison of observations to the Hurley et al. (2005) M67 simulation (4.5 Gyr). Our combined WIYN $3.5 \mathrm{~m}$ and DAO NGC $188 \mathrm{RV}$ data set covers a magnitude range of $12 \leqslant \mathrm{~V} \leqslant 16.5\left(\sim 0.9 \mathrm{M}_{\odot}\right.$ to the brightest stars in the cluster $)$, and a $1^{\circ}$ diameter region on the sky. We have thus limited the Hurley et al. (2005) M67 simulation to match this range of observations. Detailed information regarding the analysis and results of our NGC 188 observations can be found in Geller et al. (2007, in preparation). Here we simply summarize key results from this project.

\section{Results}

We have measured RVs for 1014 stars in the direction of NGC 188 with a precision of $0.4 \mathrm{~km} \mathrm{~s}^{-1}$, and have calculated RV membership probabilities for stars with $\geqslant 3$ measurements. Our careful membership determination reveals 283 single member stars and 137 member binaries, yielding an observed binary fraction of $33 \%$. We also identify 17 blue straggler (BS), 13 of which are RV variables, and 54 giant stars, 20 of which are RV variables. In the magnitude and spatially limited M67 simulation, we find 137 single stars and 249 RV variables, yielding a binary fraction of $65 \%$. We also find 17 BS stars, 6 of which are RV variables, and 30 giants, 15 of which are RV variables. We analyze these stellar populations in the following figures. 


\subsection{The Hard Binary Population}
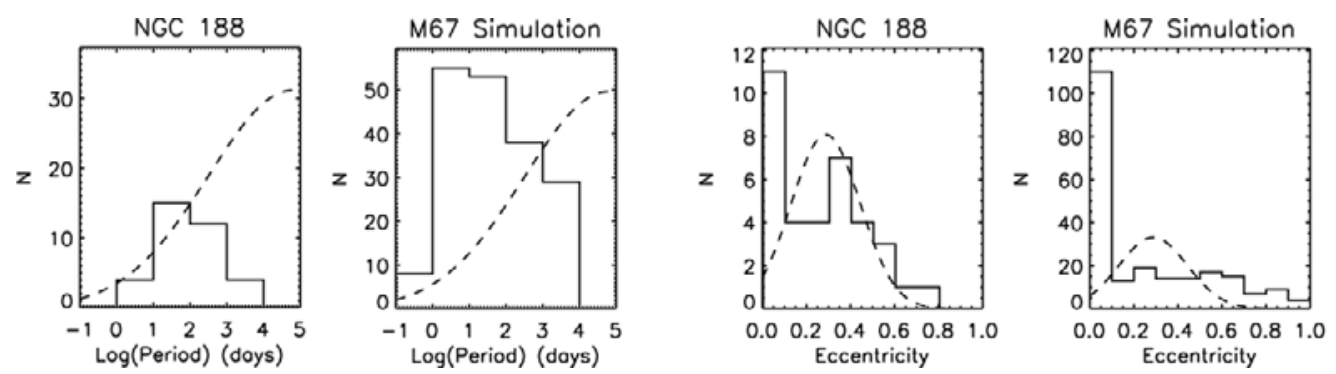

Figure 1. We show the main-sequence binary period (left) and eccentricity (right) distributions for NGC 188 and the M67 simulation in histogram form. We have also over-plotted the Duquennoy \& Mayor (1991) field binary distributions normalized to each cluster as a fiducial line. The hard-soft boundary in these clusters is on the order of $10^{4}$ days. The binary population of the M67 simulation is markedly different from the NGC 188 binary population as well as the field. Note the decrease in binaries with periods $>100$ days in the model, possibly due to dynamical interactions within the open cluster. Currently we observe a similar departure from the field distribution in this period range in NGC 188. However, we do not yet know if this is due to dynamics or incompleteness in our sample. The M67 model eccentricity distribution shows a much larger fraction of circular binaries than NGC 188, and the distribution follows a much different shape than both NGC 188 and the field binary population.

\subsection{Spatial Distribution}
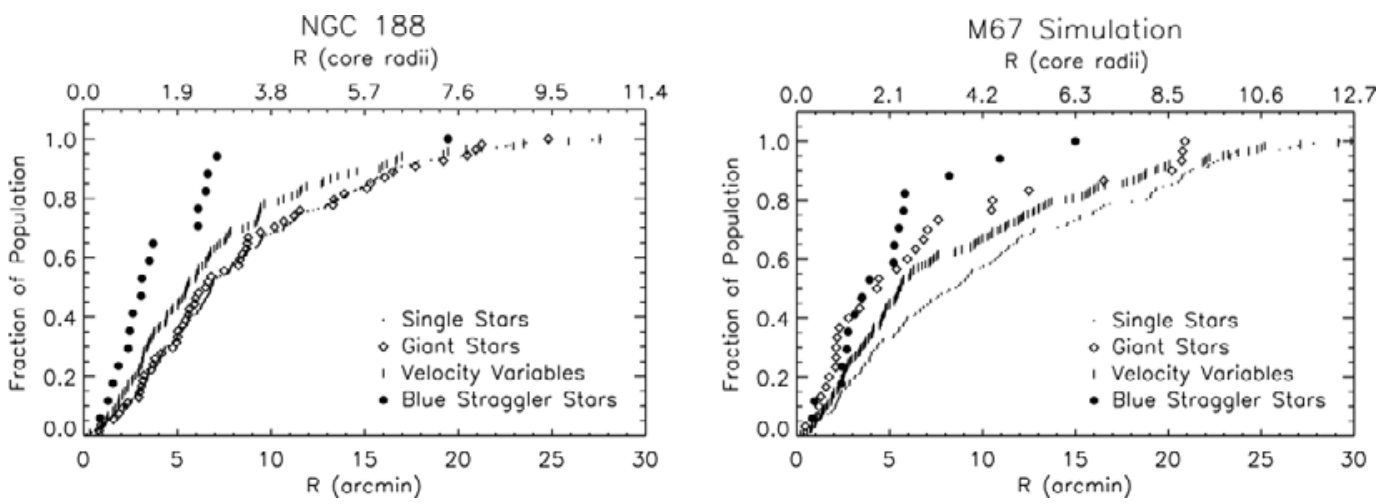

Figure 2. We plot the spatial distributions of member star populations in NGC 188 and the M67 model. We have limited to M67 simulation to include the same mass range of stars as observed in NGC 188. The single, BS, giant, and RV variable star populations are compared. For NGC 188 we conclude that both the BS stars and binaries are centrally concentrated, providing solid evidence for mass segregation. In the M67 simulation, we find the giants $\left(\bar{M} \sim 1.37 \mathrm{M}_{\odot}\right)$, BS stars $\left(\bar{M} \sim 1.56 \mathrm{M}_{\odot}\right)$, and $\mathrm{RV}$ variables $\left(\bar{M} \sim 1.68 \mathrm{M}_{\odot}\right)$ to be centrally concentrated. The M67 model average single star mass is $\sim 1.17 \mathrm{M}_{\odot}$. This central concentration is evidence for mass segregation in the M67 model. However, it is not clear why the giant stars do not appear centrally concentrated in NGC 188 while they do in the M67 model.

\section{Acknowledgements}

This work was funded by the Wisconsin Space Grant Consortium and the National Science Foundation grant number AST-0406615.

\section{References}

Duquennoy, A. \& Mayor, M. 1991, A\&A, 248, 485

Hurley, J. R., Pols, O. R., Aarseth, S. J., \& Tout, C. A. 2005, MNRAS, 363, 293 